\title{
Influence of ABO Blood Groups on Plasmodium falciparum Parasitaemia and Malaria Clinical Types in Outpatients in a Government Hospital of Douala, Cameroon
}

\author{
Thomas Kuete $^{1^{*}}$, Guy Pascal Ngaba ${ }^{1,2}$, Eric Koagne Kue ${ }^{1}$, Edouard Herve Moby Mpah ${ }^{1,2}$, and Albert Same Ekobo ${ }^{2,3}$ \\ ${ }^{1}$ Faculty of Medicine and Pharmaceutical Sciences, University of Douala, Cameroon \\ ${ }^{2}$ Bonassama district hospital, Douala, Cameroon \\ ${ }^{3}$ Yaoundé University Hospital Centre, Cameroon
}

\begin{abstract}
Falciparum malaria is a major cause of morbidity and mortality worldwide. Plasmodium infected patients carry a wide range of parasitic loads and exhibit asymptomatic, mild or severe malaria. Among host intrinsic factors which likely influence development of malaria type, controversies remain on the relationship between malaria infection and $\mathrm{ABO}$ blood groups types. This cross-sectional study was designed to investigate any relationship between ABO blood types, Plasmodium loads and clinical type of malaria among outpatients received in Bonassama hospital.
\end{abstract}

Each outpatient who volunteered for the study was examined, tested for $\mathrm{ABO}$ blood types and malaria parasites carriage. Data were statistically analyzed for any association.

Of 375 Plasmodium falciparum infected patients included, ABO blood group frequency was $45.3 \%(O), 25.3 \%$ (A), $21.6 \%$ (B), $7.8 \%$ (AB). All ABO blood groups harboured predominantly light intensities of falciparum infections; however high intensities of infections were significantly frequent in blood group A. Gender did not significantly influence $P$. falciparum infection prevalence. Infection prevalence was significantly high in less than 15 years blood group A patients. $P$. falciparum infection prevalence was not significantly different among blood group patients $(p>0.05)$. Although all $A B O$ blood group patients exhibit different clinical malaria types, severe-like malaria symptoms were exhibited more frequently among blood group $A$ and group $B$ patients. Blood group $O$ patients exhibited predominantly asymptomatic and uncomplicated malaria.

No significant association was found between Plasmodium loads, gender and age groups. Under five year blood groups $A$ and $B$ patients were likely more affected by severe malaria whereas blood group $O$ patients suffered predominantly from mild and symptomless malaria.

Findings from this study demonstrated that in Douala, young blood group A and B patients were more predisposed to high intensities of $P$. falciparum infections and severe malaria whereas blood group $O$ exhibited mostly light infections associated to mild and asymptomatic malaria.

Keywords: Falciparum malaria; ABO blood groups; Relationship; Parasitaemia; Clinical stages

\section{Introduction}

Malaria is a highly life-threatening parasitic disease caused by inhabiting red blood cells parasites named Plasmodium sp. where they multiply often lead to the burst of the host cell. An estimated 2 billion persons were at risk of infection to malaria worldwide up to 2014 of whom $84 \%$ live in sub-Saharan Africa [1-3]. This parasitic disease is a major cause of morbidity and mortality worldwide, and the infective parasite is particularly virulent among young African children, pregnant women and all age groups travellers originating from non-endemic countries $[1,4]$. Of the five species which infect man, Plasmodium falciparum is the most deadly specie. The virulence of $P$. falciparum has been associated with the capacity of the infected RBCs to surround themselves with uninfected red blood cells leading to rosetting of cells and development of severe-like malaria [5-8]. Infected subjects can develop a wide range one or more symptoms which determine one of the above clinical stages of malaria: asymptomatic malaria, mild malaria or severe malaria. The occurrence of each other clinical form of malaria has been usually associated to some risk factors either independent or related to human. Ethnicity, parasitaemia and a history of previous clinical malaria has been demonstrated to significantly influence the outcome of WHO-defined severe falciparum malaria [4]. History of previous malaria infection is thought to enhance a partial immunity which likely downregulates the outcome of clinical malaria. Such regulation by the immune system occurs much rapidly as the infections are frequent like in stable malaria transmission, and later in teenagers in seasonal malaria transmission areas. Human intrinsic risks factors have been also pointed to influence the susceptibility to malaria and development of either malaria stage. These intrinsic factors include sickle cell trait (HbAS), $\alpha$-thalassaemia, Glucose-6-phosphate dehydrogenase (G6PD) deficiency, erythrocyte variants as well as $\mathrm{ABO}$ blood group types. These factors act either by regulating parasites loads or occurrence of clinical forms of malaria. Age and acquired immune responses have long been the most frequent human-related determinants of the host susceptibility to clinical malaria and infection. Sickle cell trait (HbAS) and $\alpha$-thalassaemia are proved to protect

*Corresponding author: Thomas Kuete, University of Douala, PO Box. 2701 Douala, Cameroon, Tel: +237696629125; E-mail: thomaskuete@hotmail.com

Received: May 19, 2016; Accepted: September 26, 2016; Published: October 03, 2016

Citation: Kuete T, Ngaba GP, Kue EK, Mpah EHM, Ekobo AS (2016) Influence of ABO Blood Groups on Plasmodium Falciparum Parasitaemia and Malaria Clinical Types in Outpatients in a Government Hospital of Douala, Cameroon. J Infect Dis Med 1: 104. doi: 10.4172/2576-1420.1000104

Copyright: ( $) 2016$ Kuete T, et al. This is an open-access article distributed under the terms of the Creative Commons Attribution License, which permits unrestricted use, distribution, and reproduction in any medium, provided the original author and source are credited. 
Citation: Kuete T, Ngaba GP, Kue EK, Mpah EHM, Ekobo AS (2016) Influence of ABO Blood Groups on Plasmodium Falciparum Parasitaemia and Malaria Clinical Types in Outpatients in a Government Hospital of Douala, Cameroon. J Infect Dis Med 1: 104. doi: 10.4172/25761420.1000104

against severe and fatal malaria but none has effect on asymptomatic parasitaemia [9]. In Mali, Fulani group with rare less 202A mutation on G6PD were shown less susceptible to malaria than the Dogon group who have a high mutation of the 202A haplotype [10].

Concerning the $\mathrm{ABO}$ blood group types, there is a controversy among studies from different countries upon association between blood group and malaria. Blood group $\mathrm{O}$ individuals were relatively protected from severe malaria than those of other blood groups [11-13]. Blood group $\mathrm{O}$ patients from Ethiopia were demonstrated less prone to severe malaria as compared to patients with other blood groups [14]. Significant associations between blood group and P. falciparum malaria have been reported from cross-sectional and case control studies in Brazil [15], Gabon [16], India [17] whereas studies in Colombia, India, Sudan and Nigeria did not find any association between malaria and ABO blood group [18-22]. Despite these controversies, non-O blood groups emerged in most of the studies as significant risk factors for life-threatening malaria through the mechanism of enhanced rosette formation [12] and then blood group A and blood group B have been demonstrated as Plasmodium co-receptor in the rosetting-forming process thus enhancing occurrence of severe malaria [8].

Due to such controversies on the relationship between malaria infections outcomes and blood groups, and also due to the fact that such data are scarce in Cameroon, this study has been undertaken with the aim to determine the relationship between $\mathrm{ABO}$ blood group, malaria parasitaemia and clinical form of malaria among different age group patients who attended an hospital in Douala city of Cameroon.

\section{Patients and Methods}

\section{Study type and place}

This was a cross-sectional study carried out from November 2013 to May 2014 at the Bonassama district hospital for recruitment of patients and the Laboratory of the Faculty of Medicine and Pharmaceutical Sciences for laboratory analysis.

\section{Ethics}

This research study was carried out in compliance with the Helsinki Declaration. The protocol of the study was approved by the Littoral Regional Public Heath Delegation in Cameroon and the Ethic Committee of the University of Douala. Patient recruitment started after we obtained a written authorization letter from the director of the Bonassama district hospital. Douala town is located in a stable malaria transmission area.

\section{Data collection}

During the study period, any outpatient who attended the Bonassama District Hospital was asked to volunteer for the study. For all patients who accepted to participate, the study protocol was read to the patient then each patient who met the study criteria was included in the study. The study inclusion criteria were as follow: i) be a Cameroonian irrespective to gender; ii) age at least one year; iii) have reside continuously in the Douala town or outskirts for at least one month; iv) not have taken any antimalarial medication within the four weeks which preceded the inclusion visit; v) not have any chronic or immunodeficient known disease; vi) sign the study inform consent. Individual informed consent was obtained from adults patients but for patients aged less than 15 years and adult patients who were unable to answer to the study questionnaire, the study protocol was read to his legal parent or guardian, then the study informed consent was obtained from the later. Each eligible patient underwent a questionnaire, a clinical examination and blood tests. Questionnaire sought to collect data regarding age, sex, residence duration in the area, and complains as fever, headache, joins pains. Blood laboratory analysis was performed for $\mathrm{ABO}$ blood group testing together malaria parasites detection.

Clinical examination: The clinical examination aimed to search for symptoms related to mild as well as severe malaria as defined by WHO guidelines $[23,24]$. The main mild malaria sign investigated was hyperthermia at the inclusion visit recorded through body temperature measurement. All malaria parasites carrying patients confirmed by laboratory analysis were considered as malaria cases and classified as "severe malaria patients" if they were positive for at least one of the severe malaria criteria as outlined by the World Health Organization $[23,24]$, "mild malaria patients" if though having clinical sign of malaria they were severe-like malaria symptom-free, or "asymptomatic" if they were malaria-like symptom-free.

Malaria parasites diagnosis: Blood Plasmodium falciparum asexual stages were detected and counted using stained thick and thin blood smears performed from patient freshly collected whole blood. Blood smears were air dried and stained with $10 \%$ Giemsa solution according to routine standards $[25,26]$. The stained smears were examined under a light microscope using $100 \times$ oil immersions by an experienced laboratory technician. Slides were cross-checked by the study investigators. Parasitaemia was calculated per 500 white blood cells (WBCs) assuming that each outpatient had a mean 7500 WBCs/ $\mu$ l of blood $[25,26]$. Parasitic loads were classified according to asexual stages load as follow: 1) light for parasitic load less than 2000 trophozoites/ $\mu$ l of blood; 2) moderate if the parasitic load ranged between 2000 and 5000 asexual stages / $\mu$ l of blood; 3) high for parasitic load over 5000 asexual stages/ $\mu$ of blood.

Blood groups testing: $\mathrm{ABO}$ blood groups were typed by agglutination using commercial antisera as previously described $[27,28]$. Two drops of whole blood were placed in two different places of a grease-free clean glass slide. A drop of antiserum for blood group A was applied to one of the blood spot and a drop of blood group B antiserum was added to the second blood spot. Each blood spot and the antiserum were mixed with a sterile discardable applicator stick. The slide was then tilted to detect for agglutination and the result recorded accordingly $[27,28]$.

Data obtained were analyzed for any association between $P$. falciparum intensity of infection, clinical stage and blood group by chisquare $\left(\chi^{2}\right)$ statistical method. Statistically significance was considered at $95 \%$ level of confidence and $P$ value less than 0.05 .

\section{Results}

A total of 375 Plasmodium falciparum infected patients were included in the study. Female patients represented $58.67 \%$ of the sample. Mean age of the patients was 25 years ( \pm 19.2 years) range (one month to 84 years). $\mathrm{ABO}$ blood groups frequency occurrence was $45.3 \%, 25.3 \%, 21.6 \%$ and $7.8 \%$ for O, A, B and AB respectively.

The overall Plasmodium infection prevalence was $37.1 \%$ as detected by microscopy examination. Mixed infection with other Plasmodium specie was not included in this study.

\section{Plasmodium infection prevalence according to ABO blood groups}

As indicated in Table 1, Plasmodium infections occurred most 
Citation: Kuete T, Ngaba GP, Kue EK, Mpah EHM, Ekobo AS (2016) Influence of ABO Blood Groups on Plasmodium Falciparum Parasitaemia and Malaria Clinical Types in Outpatients in a Government Hospital of Douala, Cameroon. J Infect Dis Med 1: 104. doi: 10.4172/25761420.1000104

Page 3 of 5

frequently in blood group $\mathrm{O}$ patients whereas blood group $\mathrm{AB}$ infected patients were less frequent. However, Plasmodium infection prevalence was not significantly different among blood group patients $\left(\chi^{2}=2.38\right.$; df3; $\mathrm{p}>0.05)$. Plasmodium infection was diagnosed in $34.1 \%, 44.2 \%$, $37 \%$, and $31 \%$ of blood group $\mathrm{O}, \mathrm{A}, \mathrm{B}$ and $\mathrm{AB}$ patients respectively. Specific infection by P. falciparum occurred in $33.5 \%, 43.1 \%, 37 \%$ and $31 \%$ of blood groups $\mathrm{O}, \mathrm{A}, \mathrm{B}$ and $\mathrm{AB}$ patients respectively. The P. malariae infections cases occurred only in blood groups $\mathrm{O}$ and $\mathrm{A}$ patients.

Among infected subjects, over 15 years old patients were more representative than younger one $(66.9 \%$ vs $33.1 \%)$. Under 5 years children represented $23 \%$ of infected patients.

Within age groups, less than 15 years blood group A patients had highest infection frequency than those over 15 years old. There was no significant difference between less than 15 years infected subjects compared to those aged over 15 years among blood group $\mathrm{O}$ patients. However, in blood groups $\mathrm{A}, \mathrm{B}$ and $\mathrm{AB}$ subjects, there appeared a significant difference in Plasmodium infection between patients over 15 years old and younger ones. Sex did not significantly influence infection by Plasmodium falciparum ( $\mathrm{p}>0.05$ ) (Table 2 ).

\section{Plasmodium falciparum asexual stage intensities of infection}

Parasitic loads recorded ranged between 375 trophozoites/ $\mu$ of blood and 315000 trophozoites/ $\mu$ l of blood. As shown in Table 3 below, all blood groups patients carried predominantly light intensities of infection. However, blood group A patients had the highest frequency of high asexual stages loads (44.4\%). There was no significant difference between $\mathrm{ABO}$ blood groups types and heavy parasitic loads carriage ( $>0.05$ ). However, blood group A and blood group $A B$ patients were likely to harbour high Plasmodium asexual stage loads than the other blood group patients.

Light and heavy infections represented $83.4 \%$ and $6.5 \%$ of Plasmodium infected subjects respectively. Moderate infection accounted for $10.1 \%$ of Plasmodium carriers. One 7 years old blood group $\mathrm{AB}$ female patient harboured more than 50000 asexual stages/ $\mu$ of blood.

Light Plasmodium falciparum asexual stage load (less than 2000 trophozoites/ $\mu \mathrm{l}$ of blood) was predominant $(76.3 \%)$ among included subjects. $26.4 \%$ of these light parasitic loads carrying patients were under 15 years old, and $73.6 \%$ of them were over 15 years old. One 7 years old patient had a parasitic load over 50000 trophozoites/ $\mu$ of blood.

\section{Clinical trends of Plasmodium falciparum carrying patients}

At inclusion, $48 \%$ of Plasmodium infected had at least a malaria-like symptom and the remaining patients were symptom-free. Among those with a malaria-like symptom, $86.7 \%$ were feverish.

Among P. falciparum infected subjects $40.3 \%$ were asymptomatic, $48.9 \%$ had uncomplicated malaria and $10.8 \%$ showed at least severelike malaria symptom. As shown in Table 3, symptom-free and mild malaria patients were most common groups in blood group $\mathrm{O}, \mathrm{B}$ and $\mathrm{AB}$ patients. Severe malaria was more frequent among blood group A patients and blood group $A B$ patients than mild and symptomless malaria. Blood group A patients were likely to develop more severe malaria symptom than the other blood groups. There was however no significant difference between blood group types and the clinical type of malaria developed by the patients $(p>0.05)$. Analysis of prevalence of clinical malaria types with respect to age groups and blood group indicated that severe malaria occurred mainly in less than 15 years infected subjects. Within blood group A and blood group B infected subjects, severe malaria occurred mainly in under 15 years subjects, whereas only under 5 years blood group $\mathrm{AB}$ suffered from complicated malaria. Both younger and older blood group $\mathrm{O}$ patients suffered from severe malaria.

Asymptomatic $P$. falciparum infections and uncomplicated malaria occurred at all age in blood groups $\mathrm{O}$, blood group $\mathrm{A}$ and blood group $\mathrm{B}$ patients. However, prevalence of asymptomatic and uncomplicated malaria was significantly higher in less than 15 years blood group A and blood group B Plasmodium infected patients than in older subjects $(\mathrm{p}<0.05)$.

\section{Discussion}

This study aimed to find any association between intensities of asexual stages Plasmodium falciparum infection, ABO blood group types and clinical features of malaria among outpatients and inpatients received at Bonassama district hospital in Cameroon. This hospital is located in Douala town which is known as area of stable malaria [29]. The recruitment period extended from March to May which corresponds to the rainy season and therefore favourable for malaria cases. Diagnostic techniques used namely thick plus thin blood smears

\begin{tabular}{|c|c|c|c|c|c|c|c|c|c|c|c|c|c|c|}
\hline \multirow{3}{*}{ Blood group } & \multirow{2}{*}{\multicolumn{4}{|c|}{ Specific infections }} & \multicolumn{4}{|c|}{ Gender } & \multicolumn{6}{|c|}{ Age (years) } \\
\hline & & & & & \multicolumn{2}{|c|}{ Male } & \multicolumn{2}{|c|}{ Female } & \multicolumn{2}{|c|}{$0-4$} & \multicolumn{2}{|c|}{$5-14$} & \multicolumn{2}{|c|}{$\geq 15$} \\
\hline & $\mathbf{N}$ & Global & P. falciparum & P. malariae & $\mathbf{N}$ & $\%$ & $\mathbf{N}$ & $\%$ & $\mathbf{N}$ & $\%$ & $\mathbf{N}$ & $\%$ & $\mathbf{N}$ & $\%$ \\
\hline $\mathrm{O}$ & 170 & 34.1 & 33.5 & 0.6 & 71 & 30.1 & 99 & 36.4 & 36 & 30.5 & 12 & 33.3 & 122 & 35.2 \\
\hline A & 95 & 44.2 & 43.1 & 1.0 & 41 & 46.3 & 54 & 42.6 & 24 & 50.0 & 12 & 75.0 & 59 & 35.6 \\
\hline B & 81 & 37.0 & 37.0 & 0.0 & 32 & 31.2 & 49 & 40.8 & 26 & 34.6 & 5 & 0.0 & 50 & 42.0 \\
\hline$A B$ & 29 & 31.0 & 31.0 & 0.0 & 11 & 27.3 & 18 & 33.3 & 4 & 0.0 & 2 & 50.0 & 23 & 34.8 \\
\hline & 375 & 37.1 & 36.5 & 0.53 & 155 & 34.8 & 220 & 38.6 & 90 & 35.5 & 31 & 45.2 & 254 & 36.6 \\
\hline
\end{tabular}

Table 1: Plasmodium infection prevalence in blood group participants according to Plasmodium species, gender and age. $\mathrm{N}=$ sample size examined.

\begin{tabular}{|c|c|c|c|c|c|c|c|}
\hline \multirow{2}{*}{$\begin{array}{l}\text { Asexual stages } / \mu l \text { of } \\
\text { blood }\end{array}$} & \multicolumn{4}{|c|}{ ABO blood groups } & \multicolumn{3}{|c|}{ Age groups (years) } \\
\hline & A & B & $A B$ & 0 & $<5$ & $5-14$ & $\geq 15$ \\
\hline $1-2000$ & 35.8 & 33.3 & 27.6 & 27.6 & 31.1 & 32.2 & 30.7 \\
\hline $2001-5000$ & 4.2 & 1.2 & 0.0 & 5.3 & 2.2 & 6.4 & 3.9 \\
\hline$>5000$ & 4.2 & 2.4 & 3.4 & 1.2 & 2.2 & 6.4 & 1.9 \\
\hline TOTAL & 44.2 & 37.0 & 31.0 & 34.1 & 35.5 & 45.2 & 36.6 \\
\hline
\end{tabular}

Table 2: Frequency of $P$. falciparum asexual stage loads according to $A B O$ blood groups and age. 
Citation: Kuete T, Ngaba GP, Kue EK, Mpah EHM, Ekobo AS (2016) Influence of ABO Blood Groups on Plasmodium Falciparum Parasitaemia and Malaria Clinical Types in Outpatients in a Government Hospital of Douala, Cameroon. J Infect Dis Med 1: 104. doi: 10.4172/25761420.1000104

Page 4 of 5

\begin{tabular}{|c|c|c|c|c|c|c|c|c|c|c|c|c|c|c|c|c|c|c|c|c|c|c|}
\hline \multirow{3}{*}{$\begin{array}{l}\text { Blood } \\
\text { group }\end{array}$} & \multicolumn{12}{|c|}{ Prevalence of clinical malaria type } & \multicolumn{10}{|c|}{ Frequency of clinical malaria type } \\
\hline & \multicolumn{4}{|c|}{ Asymptomatic } & \multicolumn{4}{|c|}{ Uncomplicated } & \multicolumn{4}{|c|}{ Severe } & \multicolumn{3}{|c|}{ Asymptomatic } & \multicolumn{3}{|c|}{ Uncomplicated } & \multicolumn{3}{|c|}{ Severe } & \multirow{2}{*}{ Total } \\
\hline & $\mathbf{N}$ & $0-4$ & $5-14$ & $\geq 15$ & $\mathbf{N}$ & $0-4$ & $5-14$ & $\geq 15$ & $\mathbf{N}$ & $0-4$ & $5-14$ & $\geq 15$ & $0-4$ & $5-14$ & $\geq 15$ & $0-4$ & $5-14$ & $\geq 15$ & $0-4$ & $5-14$ & $\geq 15$ & \\
\hline $\mathrm{O}$ & 23 & 16.7 & 33.3 & 10.6 & 31 & 30.5 & 50.0 & 11.5 & 3 & 5.5 & 0.0 & 0.8 & 4.3 & 2.9 & 9.3 & 7.9 & 4.3 & 10.1 & 1.4 & 0.0 & 0.7 & 41.0 \\
\hline A & 14 & 29.2 & 16.7 & 8.5 & 21 & 37.5 & 41.7 & 11.9 & 6 & 16.7 & 16.7 & 0.0 & 5.0 & 1.4 & 3.6 & 6.5 & 3.6 & 5.0 & 2.9 & 1.4 & 0.0 & 29.5 \\
\hline B & 18 & 11.5 & 60.0 & 24.0 & 12 & 7.7 & 0.0 & 20.0 & 4 & 11.5 & 20.0 & 0.0 & 2.1 & 2.1 & 8.6 & 1.4 & 0.0 & 7.2 & 2.1 & 0.7 & 0.0 & 24.5 \\
\hline$A B$ & 1 & 0.0 & 0.0 & 4.3 & 4 & 0.0 & 0.0 & 17.4 & 2 & 50.0 & 0.0 & 0.0 & 0.0 & 0.0 & 0.7 & 0.0 & 0.0 & 2.9 & 1.4 & 0.0 & 0.0 & 5.0 \\
\hline \multirow[t]{2}{*}{ Total } & 56 & 17.7 & 29.0 & 12.2 & 68 & 24.4 & 35.5 & 13.8 & 15 & 12.2 & 9.7 & 0.4 & 11.5 & 6.5 & 22.3 & 15.8 & & 25.2 & 7.9 & 2.1 & 0.7 & 100 \\
\hline & \multicolumn{4}{|c|}{$X^{2}=5.45 ;$ df6 } & \multicolumn{4}{|c|}{$X^{2}=11.9 ; d f 6$} & \multicolumn{4}{|c|}{$X^{2}=5.45 ; d f 6$} & \multicolumn{3}{|c|}{$X^{2}=15.01 ; d f 6$} & \multicolumn{3}{|c|}{$x^{2}=11.7 ; \mathrm{df} 6$} & \multicolumn{3}{|c|}{$X^{2}=2.08 ;$ df6 } & \\
\hline
\end{tabular}

Table 3: Prevalence and frequency of clinical malaria form according to blood group and age. $\mathrm{N}=$ Plasmodium falciparum infected cases recorded.

and Rapid Diagnostic tests were the most recommended for malaria diagnosis and better management of malaria cases $[1,25,24,30]$. ABO blood group types were determined as indicated by the manufacturer leaflet using the Beth-Vincent method which the most common approach used in health facilities in Cameroon.

The distribution of patients according to $\mathrm{ABO}$ blood group types was almost similar to the overall known distribution in the African population and elsewhere with a predominance of blood group $\mathrm{O}$ subjects $[31,32]$.

Plasmodium falciparum infections occurred in all blood groups patients. Since there appeared no significant difference in infection prevalence, the blood groups seemed not to influence infection by malaria parasites. As usually described in most epidemiological data in Cameroon and most malaria endemic areas, $P$. falciparum was the predominant specie occurring in $98.6 \%$ infected patients. $P$. falciparum infections occurred in all blood group patients whereas $P$. malariae were recorded only in blood groups $\mathrm{O}$ and A patients. Predominance of $P$. falciparum in this study corroborated with previous reports in Cameroon which pointed the latest specie to represent over $90 \%$ of the overall Plasmodium infection in this country alongside P. malariae and P. ovale [29].

Blood group $\mathrm{O}$ patients had the highest infection prevalence compared to other blood group patients. This result may be due to highest sample size of blood group $\mathrm{O}$ in the study. Blood group $\mathrm{O}$ subjects therefore were likely to be encountered by female Anopheles sp flies than other blood group subjects.

Concerning $P$. falciparum asexual stage loads, it was predominantly parasites of light intensity in all blood groups. However, frequency of light intensity of infection was higher in blood group $\mathrm{O}$ and decreased as intensity of infection became heavy. We didn't find any previous data concerning $\mathrm{ABO}$ blood group types and $P$. falciparum asexual stage loads. Heavy intensities of infection were most common among infected blood group A patients.

Concerning clinical malaria type, all blood group patients presented predominantly mild and asymptomatic malaria. However, since blood group A and blood group B infected patients presented the greater number of severe-like malaria, these blood groups likely predispose to development of severe symptom of malaria. This observation corroborates previous data from Ethiopia which reported a predominance of severe malaria among blood group A P. falciparum infected patients than other blood groups [14]. Previous studies have already pointed the $\mathrm{ABO}$ blood group type in rosetting process which is the dominant process of the pathogenesis leading to severe malaria [21]. Recent studies among in Malian children demonstrated rosetting (the well-known Plasmodium falciparum parasite virulence factor) to occur more frequently in Plasmodium falciparum parasites isolates from severe malaria patients compared with non-severe hyperparasitemia and uncomplicated malaria controls [33]. In order to identify the receptors and some mechanisms which govern the interplay between malaria parasites and blood group antigens, biochemical investigations pointed blood group A and blood group B antigens as coreceptors of Plasmodium parasites in the development of severe malaria by enhancing rosetting formation in $P$. falciparum infected red blood cells, a process which did not occur frequently with blood group $\mathrm{O}$ antigens [8]. Also, concerning the contribution of $\mathrm{ABO}$ blood group types in the development of severe malaria, rosette-forming adhesion molecules rosetting have been identified in all $\mathrm{ABO}$ blood group red blood cells with a marked preference for group A over group B, which in turn is preferred to group $\mathrm{O}$ red blood cells $[34,35]$. Observations in this study showing blood group A and blood group B P. falciparum infected to develop more frequently severe malaria than blood group $\mathrm{O}$ patients were therefore in accordance previous observations. However, since within P. falciparum infected blood group A and blood group B severe malaria symptoms occurred more frequently in younger subjects, this suggested that absence of immunity may still be a major factor which modulates development of clinical malaria in endemic areas. This high susceptibility of young patients to severe malaria confirmed their high vulnerability to malaria and call for more consideration of this age group in the malaria control strategy by the World Health Organization and malaria endemic countries health ministries in Africa [1-3,30].

Results from this study also indicated that $P$. falciparum infected blood group $\mathrm{O}$ patients developed predominantly asymptomatic and uncomplicated malaria, and less frequently severe malaria symptoms. Such data agree with previous reports which pointed blood group O Malian children to be less prone to severe malaria than other blood groups subjects [11]. This less susceptibility of blood group O to severe malaria may be explained with reports from studies from studies in pregnant women which showed significant association between blood group $\mathrm{O}$ and increased placental malaria infection in primiparae and multiparae compared to other $\mathrm{ABO}$ phenotypes, a process which may modulate the pathogenesis of malaria in offspring [35]. A recent study in primiparae living in an area of high malaria endemicity of Ghana also suggested the protective influence of blood group $\mathrm{O}$ to severe malaria against $P$. falciparum infection [13].

\section{Conclusion}

Data from this study indicated that all $\mathrm{ABO}$ blood groups were likely to be infected by Plasmodium and developed asymptomatic, uncomplicated as well as severe malaria. However, blood group $\mathrm{O}$ infected subjects carried mostly light infections intensities and developed more frequently asymptomatic and mild malaria whereas severe malaria was exhibited more frequently by young blood group A and blood group B infected patients. The study emphasized that occurrence of heavy intensities of $P$. falciparum infections and high 
Citation: Kuete T, Ngaba GP, Kue EK, Mpah EHM, Ekobo AS (2016) Influence of ABO Blood Groups on Plasmodium Falciparum Parasitaemia and Malaria Clinical Types in Outpatients in a Government Hospital of Douala, Cameroon. J Infect Dis Med 1: 104. doi: 10.4172/25761420.1000104

frequency of severe malaria recorded in blood group A and blood group B patients were more related to young age of patients.

\section{Conflict of Interests}

The authors of this manuscript declare that they have no conflict of interest concerning this study.

\section{Acknowledgements}

The authors of this research are very grateful to the study participants for their cooperation and the health management and working staff of Bonassama district hospital. We also sincerely thank the staff of the parasitology laboratory of the Faculty of Medicine and Pharmaceutical Sciences of the University of Douala for their involvement in data collection.

\section{Contribution of Authors}

ASE and TK designed the study and were involved in all aspects of the study, data collection, analysis, interpretation and in writing of the manuscript. EKK, GPN, EHMM participated in data collection. ASE supervised the study. All authors read and approved the manuscript.

\section{References}

1. World Health Organization (2013) World Malaria report 2013.

2. World Health Organization (2014) World Malaria report 2014.

3. World Health Organization (2015) World Malaria report 2015.

4. Phillips A, Bassett P, Szeki S, Newman S, Pasvol G (2009) Risk factors for severe disease in adults with falciparum malaria. Clin Infect Dis 48: 871-878.

5. Carlson J, Helmby H, Hill AVS, Brewster D, Greenwood BM, et al. (1990) Human cerebral malaria: association with erythrocyte rosetting and lack of antirosetting antibodies. Lancet 336: 1457-1460

6. Carlson J, Wahlgren M (1992) Plasmodium falciparum erythrocyte rosetting is mediated by promiscuous lectin-like interactions. J Exp Med 176: 1311-1317.

7. Ringwald P, Peyron F, Lepers JP, Rabarison P, Rakotomalala C, et al. (1993) Parasite virulence factors during falciparum malaria: rosetting, cytoadherence, and modulation of cytoadherence by cytokines. Infect Immun 61: 5198-5204.

8. Barragan A, Kremsner PG, Wahlgren M, Carlson J (2000) Blood group A antigen is a coreceptor in Plasmodium falciparum rosetting. Infect Immun 68: 2971-2975.

9. Williams TN (2006) Human red blood cell polymorphisms and malaria. Curr Opin Microbiol 9: 388-94.

10. Maiga B, Dolo A, Campino S, Sepulveda N, Corran P, et al. (2014) Glucose-6phosphate dehydrogenase polymorphisms and susceptibility to mild malaria in Dogon and Fulani, Mali. Malaria J 13: 270.

11. Rowe JA, Handel IG, Thera MA, Deans AM, Like KE, et al. (2007) Blood group $O$ protects against severe Plasmodium falciparum malaria through the mechanism of reduced rosetting. Proc Natl Acad Sci USA 104: 17471-17476.

12. Rowe JA, Opi DH, Williams TN (2009) Blood groups and malaria: fresh insights into pathogenesis and identification of targets for intervention. Curr Opin Hematol 16: 480-487

13. Bedu-Addo G, Gai PP, Meese S, Eggelte TA et al. (2014) Reduced prevalence of placental malaria in primiparae with blood group O. Malaria J 13: 289.

14. Tekeste Z, Petros B (2010) The ABO blood group and Plasmodium falciparum malaria in Awash, Metehara and Ziway areas, Ethiopia. Malaria J 9: 280

15. Beiguelman B, Alves FP, Moura MM, Engracia V, Nunes AC, et al. (2003) The association of genetic markers and malaria infection in the Brazilian Western Amazonian region. Mem Inst Oswaldo Cruz 98: 455-460.
16. Migot-Nabias F, Mombo LE, Luty AJ, Dubois B, Nabias R, et al. (2000) Human genetic factors related to susceptibility to mild malaria in Gabon. Genes Immun 1: 435-441.

17. Pant CS, Gupta DK, Sharma RC, Gautam AS, Bhatt RM (1992) Frequency of $A B O$ blood groups, sickle cell haemoglobin, G-6-PD deficiency and their relation with malaria in scheduled castes and scheduled tribes of Kheda District, Gujarat. Indian J Malariol 29: 235-239.

18. Martin SK, Miller LH, Hicks CU, David-West A, Ugbode C, et al. (1979) Frequency of blood group antigens in Nigerian children with falciparum malaria. Trans R Soc Trop Med Hyg 73: 216-218.

19. Kassim OO, Ejezie GC (1982)ABO blood groups in malaria and schistosomiasis haematobium. Acta Trop 39: 179-184.

20. Bayoumi RA, Bashir AH, Abdulhadi NH (1986) Resistance to falciparum malaria among adults in central Sudan. Am J Trop Med Hyg 35: 45-55.

21. Thakur A, Verma IC (1992) Malaria and ABO blood groups. Indian J Malario 29: 241-244.

22. Montoya F, Restrepo M, Montoya AE, Rojas W (1994) Blood groups and malaria. Rev Inst Med Trop Sao Paulo 36: 33-38.

23. World Health Organization (2000) Severe falciparum malaria: World Health Organization, Communicable Diseases Cluster. Trans R Soc Trop Med Hyg 94: 1-90.

24. World Health Organization (2002) Management of severe malaria Practical Hand Book ( $2^{\text {nd }}$ edn)

25. Cheesebrough M (1998) District laboratory practice in tropical countries Cambridge: Cambridge University press.

26. World Health Organization (2015) Microscopy for the detection, identification and quantification of malaria parasites on stained thick and thin blood films in research settings: Procedure, Manual methods.

27. Beth Vincent (1918) A rapid macroscopic agglutination test for blood groups, and its value in testing donors for transfusion. JAMA 70: 1219-1220.

28. Godet M, Chevillotte J (2013) ABO compatibility testing with the Beth Vincent test. Rev Infirm 196: 53-54.

29. Mouchet J, Carnevale $P$, Coosemans $M$, Fontenille $D$, Ravaonjanahary $C$, et al. (1993) Typology of malaria in Africa. Cahiers Santé 3: 220-238.

30. World Health Organization (2013) World Malaria Report 2012.

31. Reid RE, Lomas-Francis C (2004) The blood group antigen ( $2^{\text {nd }}$ edn). Elsevier Academic Press.

32. Geoff DS (2013) Human blood Groups ( $3^{\text {rd }}$ edn). Wiley-Black.

33. Doumbo OK, Thera MA, Kone AK, Raza A, Tempest LJ, et al. (2009) High levels of Plasmodium falciparum rosetting in all clinical forms of severe malaria in African children. Am J Trop Med Hyg 81: 987-993.

34. Vigan-Womas I, Guillotte M, Juillerat A, Hessel A, Raynal B, et al. (2012) Structural basis for the ABO blood-group dependence of Plasmodium falciparum rosetting. PLoS Pathog 8: e1002781.

35. Loscertales MP, Brabin BJ (2006) ABO phenotypes and malaria related outcomes in mothers and babies in The Gambia: a role for histo-blood groups in placental malaria? Malaria J 5: 1-6. 